\title{
Affine Almost Automorphic Actions on Compact Nilmanifolds
}

\author{
S.G. Dani, Riddhi Shah \& Puneet Sharma
}

November 11, 2018

\begin{abstract}
We discuss conditions under which an affine automorphism of a compact nilmanifold is almost automorphic, and the structure of such automorphisms from a dynamical point of view.
\end{abstract}

\section{Introduction}

Let $X$ be a compact second countable space and $\alpha$ a homeomorphism of $X$. A point $x \in X$ is said to be almost automorphic for the action of $\alpha$ if for any $x, y \in X$ and any sequence $\left\{k_{i}\right\}$ in $\mathbb{Z}$ such that $\alpha^{k_{i}}(x) \rightarrow y$ we have $\alpha^{-k_{i}}(y) \rightarrow x$. The action of $\alpha$ on $X$ is said to be almost automorphic if every $x \in X$ is almost automorphic for the $\alpha$-action. It may be expected that such a behaviour would be rare. In this note we discuss the notion for affine automorphisms of compact nilmanifolds; the results illustrate the restrictive nature of the condition.

Let $N$ be a simply connected nilpotent (Lie) group and $\Gamma$ be a lattice in $N$ (i.e. $\Gamma$ is a discrete co-compact subgroup in $N$ ). Then $N / \Gamma$ is a compact manifold, and the quotients arising in this way are called nilmanifolds. By an automorphism $A$ of $N / \Gamma$ we mean a self-map such that $A(g \Gamma)=\tilde{A}(g) \Gamma$ for all $g \in N$, where $\tilde{A}$ is a (Lie) automorphism of $N$ leaving $\Gamma$ invariant (viz. such that $\tilde{A}(\Gamma)=\Gamma$ ). For $a \in N$, the map $T_{a}: N / \Gamma \rightarrow N / \Gamma$ given by $g \Gamma \mapsto$ $a g \Gamma$, for all $g \in G$, is called the translation by $a$. A transformation of $N / \Gamma$ of the form $T=T_{a} \circ A$ where $a \in N$ and $A$ is an automorphism of $N / \Gamma$ is called affine automorphism of $N / \Gamma$. An automorphism $\tilde{A}$ of $N$ is said to be 
unipotent if the corresponding automorphism of the Lie algebra is a unipotent linear transformation, and we call an automorphism $A$ of $N / \Gamma$ unipotent if it is induced by a unipotent automorphism $\tilde{U}$ such that $\tilde{U}(\Gamma)=\Gamma$.

Our aim is to characterize affine automorphisms of compact nilmanifolds which are almost automorphic. It turns out that if $T=T_{a} \circ A$ is almost automorphic then for some $r \in \mathbb{N}, A^{r}$ is unipotent (see Proposition 6.1, and the remark preceding it). We note also that for a transformation $T, T^{r}$ is almost automorphic, for $r \in \mathbb{N}$, if and only if $T$ is almost automorphic. Since $T^{r}$ is of the form $T_{a_{r}} \circ A^{r}$, for some $a_{r} \in N$, this shows that it suffices to consider affine automorphisms $T=T_{a} \circ U$, with $U$ unipotent, and in the interest of simplicity of exposition, until $\S 6$ we shall restrict to this situation.

We prove the following.

Theorem 1.1. Let $N$ be a simply connected nilpotent Lie group and $\Gamma$ be a lattice in $N$. Let $T=T_{a} \circ U$ be an affine automorphism of $N / \Gamma$, where $U$ is a unipotent automorphism of $N / \Gamma$. Let $\tilde{U}$ be the automorphism of $N$ corresponding to $U$. The point $\Gamma$ is almost automorphic for the $T$-action if and only if there exists a closed abelian subgroup $A$ of $N$ such that $A \Gamma$ is closed, $a \in A$ and $\tilde{U}(g)=g$ for all $g \in A$; in particular, if $\Gamma$ is almost automorphic then $\tilde{U}(a)=a$.

The theorem implies the following towards unipotent affine automorphisms being almost automorphic.

Corollary 1.2. Let the notation be as in Theorem 1.1. Then $T$ is almost automorphic if and only if there exists a closed connected abelian subgroup $A$ of $N$ such that $A \Gamma$ is closed, $x^{-1} a \tilde{U}(x) \in A$ for all $x \in N$ and $\tilde{U}(g)=g$ for all $g \in A$. In particular, if $T$ is minimal and almost automorphic then $N$ is abelian and $T$ is a translation of the torus $N / \Gamma$.

The "if" part is straightforward in both Theorem 1.1 and Corollary 1.2 (see $\$ 4$ for details). The main point of the results is that the converse also holds.

The conclusion of the corollary means that, from the point of view of dynamics, almost automorphic affine transformations of compact nilmanifolds consist of a collection of translations of tori put together; the "putting together" would of course depend on the nilmanifold and the transformation.

For translations we get the following characterisation. 
Corollary 1.3. Let $N$ be a simply connected nilpotent Lie group and $\Gamma$ be a lattice in $N$. Let $a \in N$. Then the translation $T_{a}$ of $N / \Gamma$ is almost automorphic if and only if there exists a closed connected abelian normal subgroup $A$ of $N$ such that $A \Gamma$ is closed.

The following describes certain simple necessary conditions, at the "infinitesimal" (or Lie algebra) level, on the automorphism and the translating element for an affine automorphism of a compact nilmanifold to be almost automorphic.

Corollary 1.4. Let the notation be as in Theorem 1.1. Furthermore let $\mathfrak{N}$ be

the Lie algebra of $N, \mathfrak{U}$ be the Lie automorphism of $\mathfrak{N}$ corresponding to $\tilde{U}$, and $\operatorname{Ad}_{a}$ be the adjoint transformation of $\mathfrak{N}$ corresponding to $a$. Let $I$ and 0 denote the identity and zero transformations of $\mathfrak{N}$, respectively. Suppose $T$ is almost automorphic. Then the following holds:

i) $(\mathfrak{U}-I)\left(\operatorname{Ad}_{a} \circ \mathfrak{U}-I\right)=0$; in particular, if $T$ is an automorphism (viz. a is the identity) then $\tilde{U}$ has nilrank at most 1 , namely $(\tilde{U}-I)^{2}=0$.

ii) The image of $\operatorname{Ad}_{a} \circ \mathfrak{U}-I$ is an abelian Lie subalgebra.

As we shall see from an example these conditions are not sufficient for the converse to hold, even in the case of automorphisms.

\section{Minimal Translations}

In this section we shall prove the following proposition which in particular proves the statement of Theorem 1.1 in the special case of minimal translations. We recall that an action is said to be minimal if there is no proper nonempty closed invariant subset, or equivalently if every orbit is dense.

Proposition 2.1. Let $N$ be a simply connected nilpotent Lie group and $\Gamma$ be a lattice in $N$. Let $\Phi=\left\{a_{t}\right\}$ be a one-parameter subgroup of $N$. Suppose that the action of $\Phi$ on $N / \Gamma$ is minimal, and that $\Gamma$ is an almost automorphic point of the action. Then $N$ is abelian.

Proof. We proceed by induction on the dimension $n$ of $N$. The assertion is evident for $n=1$. Now suppose the dimension is $n$ and that the proposed statement holds when the dimension is less than $n$. 
Let $Z$ denote the centre of $N$. Then $Z$ is topologically isomorphic to $\mathbb{R}^{k}$ for some $k$ and $Z \cap \Gamma$ is a lattice in $Z$. Let $P$ be a one-parameter subgroup of $Z$ containing a nontrivial element of $\Gamma$; we note that $P \Gamma$ is a subgroup, and as $P /(P \cap \Gamma)$ is compact, $P \Gamma$ is closed. Now, $N / P$ is a simply connected nilpotent Lie group and $\Gamma P / P$ is a lattice in $N / P$. The $\Phi$-action on $N / \Gamma$ factors to an action on $(N / P) /(\Gamma P / P)$ which is minimal and almost automorphic. By the induction hypothesis therefore $N / P$ is abelian.

Now suppose, if possible, that $N$ is nonabelian. Let $\mathfrak{N}$ be the Lie algebra of $N$ and $\exp : \mathfrak{N} \rightarrow N$ be the exponential map. Let $\Lambda$ be the (additive) subgroup of $\mathfrak{N}$ generated by $\exp ^{-1}(\Gamma)$. Then $\Lambda$ is a lattice in $\mathfrak{N}$ and $\exp (\Lambda)$ is a lattice in $N$; (see [3], Theorem 2.12, addendum to the main statement). Clearly $\exp (\Lambda)$ contains $\Gamma$ as a subgroup of finite index. Moreover, since $\Gamma$ is $\tilde{U}$-invariant, so is $\exp (\Lambda)$, and the factor of $T$ on $N / \exp (\Lambda)$ is almost automorphic and minimal. Therefore, in the proof of the theorem replacing $\Gamma$ by $\exp (\Lambda)$ and modifying notation we may assume that $\Gamma=\exp (\Lambda)$, with $\Lambda$ a lattice in $\mathfrak{N}$. Now let $\mathfrak{P}$ be the (one-dimensional) Lie subalgebra corresponding to $P$. We note that $\mathfrak{P} \cap \Lambda$ is an infinite cyclic subgroup and choose a generator $e_{0}$. Then $e_{0}$ can be extended to a basis of $\Lambda$, as a free abelian group, say $\left\{e_{0}, e_{1}, \ldots, e_{d}\right\}$. Let $W$ be the subspace of $\mathfrak{N}$ spanned by $\left\{e_{1}, \ldots, e_{d}\right\}$. Since $N / P$ is abelian, $\mathfrak{N}$ can be realised as $W+\mathbb{R}$ with the Lie bracket operation given by $\left[\left(w_{1}, t_{1}\right),\left(w_{2}, t_{2}\right)\right]=$ $\left[0, \alpha\left(w_{1}, w_{2}\right)\right]$ for all $w_{1}, w_{2} \in W$ and $t_{1}, t_{2} \in \mathbb{R}$, where $\alpha$ is a bilinear form on $W$. Correspondingly $N$ can realised as $W \times \mathbb{R}$, with the multiplication given by $\left(w_{1}, t_{1}\right) \cdot\left(w_{2}, t_{2}\right)=\left(w_{1}+w_{2}, t_{1}+t_{2}+\mu\left(w_{1}, w_{2}\right)\right)$, for all $w_{1}, w_{2} \in W, t_{1}, t_{2} \in \mathbb{R}$, with $\mu$ a bilinear form on $W$, and the exponential map exp $: \mathfrak{N} \rightarrow N$ is given by $\exp ((w, t))=\left(w, \frac{1}{2} \mu(w, w)+t\right)$ for all $w \in W$ and $t \in \mathbb{R}$.

Let $\Delta$ be the integral lattice in $W$ with respect to the basis $\left\{e_{1}, \ldots, e_{d}\right\}$. Then $\Delta$ is the image of $\Gamma$ when $W$ is viewed as $N / P$, and we note also that for any $\delta \in \Delta$, as a subset of $\mathfrak{N}$, we have $\exp (\delta)=\left(\delta, \frac{1}{2} \mu(\delta, \delta)\right) \in \Gamma$.

Let

$$
F=\left\{u \in W \mid u=\sum_{i=1}^{d} \alpha_{i} e_{i}, \text { with }\left|\alpha_{i}\right| \leq \frac{1}{2} \text { for all } i\right\} .
$$

Consider any $w \in W$. Then there exist $u \in F$ and $\delta \in \Delta$ such that $w=u+\delta$. Then in $N$, we have $(w, 0)=\left(u,-\mu(u, \delta)-\frac{1}{2} \mu(\delta, \delta)\right) \cdot\left(\delta, \frac{1}{2} \mu(\delta, \delta)\right)$, and since $\left(\delta, \frac{1}{2} \mu(\delta, \delta)\right) \in \Gamma$ we get that $(w, 0) \Gamma=\left(u,-\mu(u, \delta)-\frac{1}{2} \mu(\delta, \delta)\right) \Gamma$. For $w \in W$ such that $u$ is in the interior of $F$ the $\delta$ as above is unique and in this case we shall 
denote the element $\left(-\mu(u, \delta)-\frac{1}{2} \mu(\delta, \delta)\right)$ by $\rho(w)$; hence $(w, 0) \Gamma=(u, \rho(w)) \Gamma$. We note also that given such a $w$, we have $(-w, 0)=(-u,-\mu(-u,-\delta)-$ $\left.\left.\left.\frac{1}{2} \mu(-\delta,-\delta)\right)\right) \cdot\left(-\delta, \frac{1}{2} \mu(-\delta,-\delta)\right)\right)$, so $\left.\left.\rho(-w)=-\mu(-u,-\delta)-\frac{1}{2} \mu(-\delta,-\delta)\right)\right)=\rho(w)$.

Since the $\Phi$-action is minimal it follows that there exists $r \in \mathbb{R}$ such that $T_{a_{r}}$ is minimal; this may be seen from Parry's theorem [2] together with the fact that it is true for tori. Let $(b, s)$, with $b \in W$ and $s \in \mathbb{R}$ be the pair corresponding to $a_{r} \in \Phi \subset N$, and let $T=T_{a_{r}}$. Then for any $w \in W$ and $t \in \mathbb{R}$ we have $T((w, t) \Gamma)=(b+w, s+t+\mu(b, w)) \Gamma$. Recursively we see that

$$
T^{k}((w, t) \Gamma)=\left(k b+w, k s+t+\sum_{j=0}^{k-1} \mu(b, j b+w)\right) \Gamma
$$

for all $k \geq 1$. Also, for all $w \in W$ and $t \in \mathbb{R}$ we have $T^{-1}((w, t) \Gamma)=(-b+$ $w,-s+t-\mu(b,-b+w)) \Gamma$ and recursively,

$$
T^{-k}((w, t) \Gamma)=\left(-k b+w,-k s+t-\sum_{j=1}^{k} \mu(b,-j b+w)\right) \Gamma
$$

for all $k \geq 1$.

Let $\xi \in \mathbb{R}$ be irrational. We now choose a sequence $\left\{k_{i}\right\}$ in $\mathbb{N}$ such that $T^{k_{i}}(\Gamma) \rightarrow(0, \xi) \Gamma$, as follows. Let $S$ be the translation $T_{(b, 0)}$ of $N / \Gamma$ and $\tau$ be the (cartesian) product transformation $S \times T_{\mu(b, b)}$ of $(N / \Gamma) \times(\mathbb{R} / \mathbb{Z})$; viz $\tau$ consists of translations by $(b, 0)$ and $\mu(b, b)$ in the respective components. If $\tau$ is minimal, then we can choose $\left\{k_{i}\right\}$ such that $S^{k_{i}}(\Gamma) \rightarrow(0, \xi) \Gamma$ and $k_{i} \mu(b, b) \rightarrow 0 \bmod \mathbb{Z}$. Now suppose $\tau$ is not minimal. We note that since the factors of $S$ and $T$ on $W / \Delta$ coincide, by Parry's theorem [2] $S$ is minimal. In this case we choose a $\left\{k_{i}\right\}$ such that $S^{k_{i}}(\Gamma) \rightarrow(0, \xi) \Gamma$. In either case, passing to a subsequence we shall also assume that $\left(0, k_{i} s\right) \Gamma \rightarrow(0, \sigma)$ as $i \rightarrow \infty$, where $\sigma \in \mathbb{R}$.

We note the following in the case when $\tau$ is not minimal. Under this condition, $T_{b} \times T_{\mu(b, b)}$ of $(W / \Delta) \times(\mathbb{R} / \mathbb{Z})$ is also not minimal. This further implies that there exist $p \mathbb{Z}, q \in \mathbb{N}$, and a linear form $\psi$ on $W$ such that $\psi(\Delta) \subset \mathbb{Z}$ and $\mu(b, b)=(\psi(b)+p) / q$. Since $S^{k_{i}}(\Gamma) \rightarrow(0, \xi) \Gamma,\left\{T_{b}^{k_{i}}(0)+\Delta\right\}$ which is the same as $\left\{k_{i} b+\Delta\right\}$ converges to the identity element in $W / \Delta$. Since $\mu(b, b)=(\psi(b)+p) / q$, with $\psi$ and $p, q$ as above, it follows that every limit point of $\left\{k_{i} \mu(b, b)+\mathbb{Z}\right\}$ in $\mathbb{R} / Z$ is a $q$ th root of unity, and passing to a subsequence we may assume that $\left\{k_{i} \mu(b, b)+\mathbb{Z}\right\}$ converges to a root of unity. 
Thus in either of the cases, we may assume $k_{i} \mu(b, b)+\mathbb{Z} \rightarrow \eta+\mathbb{Z}$, with $\eta$ rational.

Now, for all $i$ we have, $T^{k_{i}}(\Gamma)=\left(k_{i} b, k_{i} s+\theta_{i}\right) \Gamma$, and $S^{k_{i}}(\Gamma)=\left(k_{i} b, \theta_{i}\right) \Gamma$, where $\left.\theta_{i}=\sum_{j=0}^{k_{i}-1} \mu(b, j b)\right)=\frac{1}{2}\left(k_{i}-1\right) k_{i} \mu(b, b)$. Let $u_{i} \in F$ and $\rho_{i} \in \mathbb{R}$ be such that $\left(k_{i} b, 0\right) \Gamma=\left(u_{i}, \rho_{i}\right) \Gamma$. Then for all $i, T^{k_{i}}(\Gamma)=\left(u_{i}, k_{i} s+\rho_{i}+\theta_{i}\right) \Gamma$ and $S^{k_{i}}(\Gamma)=\left(u_{i}, \rho_{i}+\theta_{i}\right) \Gamma$, and since the latter sequence converges to $(0, \xi) \Gamma$ we get that $u_{i} \rightarrow 0$ and $\rho_{i}+\theta_{i} \rightarrow \xi \bmod \mathbb{Z}$, as $i \rightarrow \infty$. Since $\left(0, k_{i} s\right) \Gamma \rightarrow(0, \sigma)$ as $i \rightarrow \infty$ this shows that $T^{k_{i}}(\Gamma) \rightarrow(0, \sigma+\xi) \Gamma$, as $i \rightarrow \infty$.

On the other hand, for all $i, T^{-k_{i}}(\Gamma)=\left(-k_{i} b,-k_{i} s+\theta_{i}+k_{i} \mu(b, b)\right) \Gamma$. Since $u_{i} \rightarrow 0$, for all large $i$ we have $\rho_{i}=\rho\left(k_{i} b\right)$, and since $\rho\left(k_{i} b\right)=\rho\left(-k_{i} b\right)$ we get that $\left(-k_{i} b, 0\right) \Gamma=\left(-u_{i}, \rho_{i}\right) \Gamma$ for all large $i$. Hence $T^{-k_{i}}(\Gamma)=\left(-u_{i},-k_{i} s+\rho_{i}+\right.$ $\left.\theta_{i}+k_{i} \mu(b, b)\right) \Gamma$, for all $i$. Using the convergences as above we see from this that as $i \rightarrow \infty, T^{-k_{i}}(\Gamma) \rightarrow(0,-\sigma+\xi+\eta) \Gamma$. Hence $T^{-k_{i}}((0, \sigma+\xi) \Gamma) \rightarrow(0,2 \xi+\eta) \Gamma$. Since $\xi$ is irrational and $\eta$ is rational $(0,2 \xi+\eta) \Gamma \neq \Gamma$; this is a contradiction to the condition that $\Gamma$ is an almost automorphic point for the $\Phi$-action. This shows that $N$ must be abelian, thus proving the proposition.

\section{Proof of Theorem 1.1 for translations}

We next prove the Theorem 1.1 for all translations. Specifically we prove the following.

Proposition 3.1. Let $N$ be a simply connected nilpotent Lie group and $\Gamma$ be a lattice in $N$. Let $\Phi=\left\{a_{t}\right\}$ be a one-parameter subgroup of $N$ and let $a=a_{t}$ for some $t \neq 0$. Then the following conditions are equivalent.

i) $\Gamma$ is almost automorphic for the action of $T_{a}$ on $N / \Gamma$;

ii) $\Gamma$ is almost automorphic for the $\Phi$-action on $N / \Gamma$;

iii) there exists a closed connected abelian subgroup $A$ of $N$ such that $\Phi$ is contained in $A$ and $A \Gamma$ is closed.

Proof. The proof of equivalence of (i) and (ii) is routine and we shall omit it.

ii) $\Longrightarrow$ iii): We proceed by induction on the dimension of $N$. This is trivial when $N$ is one-dimensional. Now consider the general case, assuming the validity in lower-dimensional cases. If the $\Phi$-action on $N / \Gamma$ is minimal then by Proposition 2.1 it follows that $N$ is abelian, as desired. Now suppose that the 
$\Phi$-action is not minimal. Let $V=N /[N, N]$ and $\Delta=\Gamma[N, N] /[N, N]$. Since the $\Phi$-action is not minimal, by Parry's theorem the factor action on $V / \Delta$ is also not minimal. This implies that there exists a proper vector subspace $W$ of $V$ containing $\Phi[N, N]$ such that $W \Delta$ is closed. Let $M$ be the Lie subgroup of $N$ containing $[N, N]$ such that $W=M /[N, N]$. Then $\Phi$ is contained in $M$ and the $\Phi$-action on $M \Gamma / \Gamma$ is almost automorphic. By the induction hypothesis there exists a closed connected abelian subgroup $A$ containing $\Phi$ such that $A \Gamma$ is closed, thus proving (iii).

iii) $\Longrightarrow \mathrm{i}$ ): When (iii) holds $A \Gamma / \Gamma$ is a closed invariant subset the restriction to which is equivalent to a translation on $A / A \cap \Gamma$ which is a torus, and in particular it is an isometry with respect to a metric. Hence $\Gamma$ is almost automorphic. This completes the proof.

\section{Proofs of the main results}

In this section we shall deduce from the case of the translations the general case of the main theorem. The main point is to realise the unipotent affine automorphisms as restrictions of translations of higher dimensional compact nilmanifolds. This corresponds to considering the "suspension" of the given affine automorphism, in the sense of dynamics. We begin by recalling the construction.

Let $N$ be a simply connected nilpotent Lie group, $\Gamma$ be a lattice in $N, U$ a unipotent automorphism of $\mathbb{N} / \Gamma$ and $\tilde{U}$ the corresponding automorphism of $N$. Then there exists a one-parameter subgroup $\Phi=\left\{\tilde{U}_{t}\right\}$ consisting of unipotent automorphisms of $N$, with $\tilde{U}=\tilde{U}_{1}$. Let $M=\Phi \cdot N$, the semidirect product of $\Phi$ and $N$ with respect to the action of $\Phi$ by automorphisms. Then $M$ is a simply connected nilpotent Lie group. Since $\tilde{U}(\Gamma)=\Gamma$, it follows that $\Delta:=$ $\left\{\tilde{U}^{n} \mid n \in \mathbb{Z}\right\} \cdot \Gamma$ is a subgroup of $M$. Furthermore $\Delta$ is a lattice in $M$. We realise $N / \Gamma$ canonically as a subset of $M / \Delta$. We note also that the $U$-action on $N / \Gamma$ is the restriction of the translation of $M / \Delta$ by the element $\tilde{U}$; for $g \in \mathbb{N}$,

$(\tilde{U} \cdot g) \Delta=\tilde{U}(g) \tilde{U} \Delta=\tilde{U}(g) \Delta$ and the latter is the same as $U(g) \Gamma$ under the realisation of $N / \Gamma$ as a subset of $M / \Delta$.

Proof of Theorem 1.1: We follow the notation as in the hypothesis and as introduced above. Suppose that $T$ is almost automorphic. We have realised $N / \Gamma$ as a subset of $M / \Delta$, such that the $U$-action on $N / \Gamma$ is the restriction of a translation 
of $M / \Delta$, by an element, specifically $\tilde{U}$ viewed as an element of $M$. Then $T$ is also the restriction of a translation, by the element $a \tilde{U} \in M$, and we note that $\Gamma$ being almost automorphic for the $T$-action implies that $\Delta$ is almost automorphic for the translation by $a \tilde{U}$. Hence there exists a closed connected abelian subgroup $B$ of $M$, containing $a \tilde{U}$, such that $B \Delta$ is closed. The image of $B \operatorname{modulo} N$ is a connected subgroup of $\Phi$ containing $\tilde{U}$ and hence it is the whole of $\Phi$. Therefore $B=\Phi \cdot A$ where $A=B \cap N$. Also since $B \Delta$ is closed and $\Phi \cap \Delta$ is a lattice in $\Phi$ it follows that $A \Gamma$ is closed. Since $a \tilde{U} \in B$ we get $a \in A$. Also any $g \in A \subset B$ commutes with $\tilde{U}$ as an element of $M$, which shows that $\tilde{U}(g)=\tilde{U} g \tilde{U}^{-1}=g$. This proves the "only if" part of the theorem. The "if" part is obvious since under the condition the $T$-orbit of $\Gamma$ is contained in $A \Gamma / \Gamma$ which is a torus, and the action is a translation of the torus and hence an isometry with respect to a metric. This proves the theorem.

Proof of Corollary 1.2: Suppose that $T$ is almost automorphic. Let $x \in N$ be arbitrary. Then $x \Gamma$ is almost automorphic for the $T$-action. We view $N / \Gamma$ as $N / x \Gamma x^{-1}$, choosing $x \Gamma$ as the base point. Then the $T$-action corresponds to $T_{a_{x}} \circ U_{x}$, where $a_{x}=a \tilde{U}(x) x^{-1}$, and $U_{x}$ is the automorphism of $N / x \Gamma x^{-1}$ induced by the automorphism $\tilde{U}_{x}$ given by $\tilde{U}_{x}(g)=x \tilde{U}(x)^{-1} \tilde{U}(g) \tilde{U}(x) x^{-1}$. Applying Theorem 1.1 we get that there exists a closed connected subgroup $A_{x}$ such that $A_{x} x \Gamma$ is closed, $a_{x} \in A_{x}$ and $\tilde{U}_{x}(g)=g$ for all $g \in A_{x}$.

Let $\mathcal{A}$ be the set of all closed connected abelian subgroups $A$ such that $A \Gamma$ is closed. Then $\mathcal{A}$ is countable; this follows from the fact that the Lie subalgebra corresponding to $A \in \mathcal{A}$ is determined by linear equations with rational coefficients. We have $x^{-1} A_{x} x \in \mathcal{A}$ for all $x \in N$ and hence it follows that there exists $A \in \mathcal{A}$ such that the set $E$ consisting of all $x$ such that $x^{-1} A_{x} x=A$ (which is closed and hence a Borel subset), has positive Haar measure. We have $a_{x} \in A_{x}$ and hence $x^{-1} a \tilde{U}(x) \in x^{-1} A_{x} x=A$ for all $x \in E$. Since the set of $x$ such that $x^{-1} a \tilde{U}(x) \in A$ is a submanifold of $N$ and $E$ has positive Haar measure, this implies that $x^{-1} a \tilde{U}(x) \in A$ for all $x \in N$.

Now consider any $x \in E$. We have $\tilde{U}_{x}(g)=x \tilde{U}(x)^{-1} \tilde{U}(g) \tilde{U}(x) x^{-1}=g$ for all $g \in A_{x}$. Thus $\tilde{U}\left(x^{-1} g x\right)=x^{-1} g x$ for all $g \in A_{x}$, and since $A=x^{-1} A_{x} x$ this means that $\tilde{U}(g)=g$ for all $g \in A$. This shows that $A$ has the desired properties and thus proves the "only if" part of the corollary.

Now suppose that there exists a subgroup $A$ as in the statement. Let $x \in N$ 
be arbitrary. Then $x A \Gamma / \Gamma$ is a closed subset of $N / \Gamma$. For any $g \in A$ we have $T(x g \Gamma)=a \tilde{U}(x g) \Gamma=a \tilde{U}(x) \tilde{U}(g) \Gamma=a \tilde{U}(x) g \Gamma$, since $\tilde{U}(g)=g$, and $a \tilde{U}(x) \in x A$ by hypothesis, so we get that $T(x g \Gamma) \in x A \Gamma / \Gamma$. Thus $x A \Gamma / \Gamma$ is $T$-invariant. Furthermore the above relation also shows that under the canonical correspondence of $x A \Gamma / \Gamma$ with $A \Gamma / \Gamma$ the restriction of $T$ to the former corresponds to the translation action by the element $x^{-1} a \tilde{U}(x)$. Now $A \Gamma / \Gamma$ is a torus and the translation is an isometry with respect to a metric on it, so the map is almost automorphic. Since this holds for every $x$ it follows that $T$ is almost automorphic.

Finally, we note that if $T$ is also minimal then $A \Gamma / \Gamma$ as above has to be the whole of $N$, which implies that $N$ is abelian, and that $T$ is a translation of $N / \Gamma$.

Proof of Corollary 1.3: The "if" part is straightforward and we omit the proof. Now suppose $T_{a}$ is automorphic. Then by Corollary 1.2 there exists a closed connected abelian subgroup $A^{\prime}$ such that $A^{\prime} \Gamma$ is closed and $x^{-1} a x \in A^{\prime}$ for all $x \in N$. Let $B$ be the smallest closed subgroup of $N$ containing $\left\{x^{-1} a x \mid x \in N\right\}$. Then $B$ is a closed normal subgroup of $N$ contained in $A^{\prime}$. Hence $\overline{B \Gamma}$ is a closed subgroup of $A^{\prime} \Gamma$. Let $A$ be the connected component of the identity in $\overline{B \Gamma}$. Then $A$ is a closed connected subgroup. Moreover, as $\overline{B \Gamma} \subset A^{\prime} \Gamma$ and the latter is a closed subgroup, $A \subset A^{\prime}$ and hence $A$ is abelian. Since $A \Gamma$ is the closed subgroup $\overline{B \Gamma}$ and $A$ is identity component, $A$ is normalised by $\Gamma$. Since $\Gamma$ is a lattice in a simply connected nilpotent Lie group this implies that $A$ is normal in $N$ (see [3], Corollary 2 of Theorem 2.3). This completes the proof.

Proof of Corollary 1.4: By Corollary 1.2, for every $x \in N, x^{-1} a \tilde{U}(x)$ and $a$ are fixed by $\tilde{U}$, and hence so is $x^{-1} a \tilde{U}(x) a^{-1}$. Given $\xi \in \mathfrak{N}$, applying this to $\exp t \xi, t \in \mathbb{R}$, in place of $x$, and differentiating with respect to $t$ we get that $\operatorname{Ad}_{a} \circ \mathfrak{U}(\xi)-\xi$ is fixed by $\mathfrak{U}$. Thus $\mathfrak{U}\left(\operatorname{Ad}_{a} \circ \mathfrak{U}(\xi)-\xi\right)=\operatorname{Ad}_{a} \circ \mathfrak{U}(\xi)-\xi$ for all $\xi \in \mathfrak{N}$, that is, $(\mathfrak{U}-I)\left(\operatorname{Ad}_{a} \circ \mathfrak{U}-I\right)=0$. This proves $(\mathrm{i})$

Also, by Corollary 1.2, the elements $x^{-1} a \tilde{U}(x) a^{-1}$ commute with each other. Let $\mathfrak{M}=\left(\operatorname{Ad}_{a} \circ \mathfrak{U}-I\right)(\mathfrak{N})$. Then $\mathfrak{M}$ is readily seen to be a Lie subalgebra of $\mathfrak{N}$. Given $\xi \in \mathfrak{M}$ it is tangent to the curve $x_{t}^{-1} a \tilde{U}\left(x_{t}\right) a^{-1}$ where $x_{t}=\exp t \xi$ for all $t \in \mathbb{R}$. Since for $\xi, \eta \in \mathfrak{M}$ the elements in the corresponding curves as above commute with each other, it follows that $\xi$ and $\eta$ commute in $\mathfrak{N}$. Hence $\mathfrak{M}$ is commutative. This proves the Corollary. 


\section{Some further consequences}

In this section we discuss some auxiliary results around the theme of the main results.

For $N=\mathbb{R}^{d}, d \geq 1$ we have the following characterisation of almost automorphic affine automorphisms, which may be compared with Corollary 1.4 .

Proposition 5.1. Let $N=\mathbb{R}^{d}, d \geq 1$, and $\Gamma=\mathbb{Z}^{d}$. Let $T=T_{a} \circ U$ be an affine automorphism of $N / \Gamma$, where $a \in N$ and $U$ is an automorphism of $N / \Gamma$ induced by a unipotent automorphism $\tilde{U}$ of $N$. Then $T$ is almost automorphic if and only if $(\tilde{U}-I)^{2}=0$ (here $I$ and 0 are the identity and zero matrices respectively) and $\tilde{U}(a)=a$.

Proof. Suppose $T$ is almost automorphic. Then by Corollaries 1.4 and $1.2(\tilde{U}-$ $I)^{2}=0$ and $\tilde{U}(a)=a$. Conversely suppose $(\tilde{U}-I)^{2}=0$ and $\tilde{U}(a)=a$. Then every $T$-orbit is contained in an orbit, say $O$, of the subgroup $\left\{x \in \mathbb{T}^{d} \mid U(x)=x\right\}$ and restricted to $O$ the $U$-action is equivalent to a translation. Hence $T$ is almost automorphic.

The following example shows that the converse of Corollary 1.4 is however not true in general, even for automorphisms; (for the affine case we already have another necessary condition, that $a$ is fixed by $\tilde{U}$, not incorporated in Corollary 1.4 focussing on the conditions at the Lie algebra level.

Example 5.2. Let $\mathfrak{N}$ be the 4-dimensional Lie algebra with a set of basis vectors $\left\{\xi_{1}, \xi_{2}, \xi_{3}, \xi_{4}\right\}$ and the Lie bracket satisfying the conditions $\left[\xi_{1}, \xi_{2}\right]=$ $\xi_{3}=-\left[\xi_{2}, \xi_{1}\right],\left[\xi_{2}, \xi_{3}\right]=\xi_{4}=-\left[\xi_{3}, \xi_{2}\right]$ and $\left[\xi_{i}, \xi_{j}\right]=0$ for $(i, j)$ different from $(1,2),(2,1),(2,3)$ and $(3,2)$. It can be seen that $\mathfrak{N}$ is a nilpotent Lie algebra. Let $\mathfrak{U}: \mathfrak{N} \rightarrow \mathfrak{N}$ be the linear map such that $\mathfrak{U}\left(\xi_{1}\right)=\xi_{1}+\xi_{2}$ and $\mathfrak{U}\left(\xi_{i}\right)=\xi_{i}$ for $i=2,3$ and 4 . It can be verified that $\mathfrak{U}$ is a Lie algebra automorphism and $(\mathfrak{U}-I)^{2}=0$. Also the image of $\mathfrak{U}-I$ is the (one-dimensional) span of $\xi_{2}$, which is an abelian Lie subalgebra. Let $N$ be the simply connected Lie group corresponding to $\mathfrak{N}$ and let $\tilde{U}$ be the Lie automorphism of $N$ corresponding to $\mathfrak{U}$. Let $\Lambda$ be the subgroup of $\mathfrak{N}$ generated by the basis $\left\{\xi_{1}, \xi_{2}, \xi_{3}, \xi_{4}\right\}$ and $\Gamma=\exp (\Lambda)$, where exp denotes the exponential map of $\mathfrak{N}$ onto $N$. Since the basis has rational structure constants it follows that $\Gamma$ is a lattice in $N$ (cf. [3], Theorem 2.12). Also, since $\Lambda$ is $\mathfrak{U}$-invariant, it follows that $\Gamma$ is $\tilde{U}$-invariant. Let $U$ be the corresponding automorphism of $N / \Gamma$. Let $M$ be the connected subgroup of 
$N$ corresponding to the Lie subalgebra spanned by $\left\{\xi_{2}, \xi_{3}, \xi_{4}\right\}$; then $M$ is also the set of fixed points of $\tilde{U}$. For all $t \in \mathbb{R}$ let $x_{t}=\exp t \xi_{1}$, and consider the subset $E=\left\{x_{t}^{-1} \tilde{U}\left(x_{t}\right) \mid t \in \mathbb{R}\right\}$ of $M$. We see that $E$ generates $M$; this may be proved by observing that in $M /[M, M]$, which may be viewed canonically as $\mathbb{R}^{2}$, the image of $E$ is an affine line. In particular $E$ is not contained in an abelian subgroup. Hence by Corollary 1.2 we get that $U$ is not almost automorphic.

The phenomenon involved in the above Example is seen in a more general form in the following proposition.

Proposition 5.3. Let $\mathfrak{N}$ be a nilpotent Lie algebra generated, as a Lie algebra, by two elements $\xi$ and $\eta$, and $\mathfrak{U}$ be a unipotent Lie automorphism of $\mathfrak{N}$ such that $\mathfrak{U}(\xi)=\xi+\eta$. Let $N$ be a simply connected nilpotent Lie group with $\mathfrak{N}$ as its Lie algebra. Let $\tilde{U}$ be the Lie automorphism of $N$ corresponding to $\mathfrak{U}$. Let $\Gamma$ be a lattice in $N$ invariant under $\tilde{U}$ and let $U$ be the automorphism of $N / \Gamma$ induced by $\tilde{U}$. If $U$ is almost automorphic then $N$ contains a $(d-1)$-dimensional abelian $\tilde{U}$-invariant normal subgroup $M$ such that $N$ is the semidirect product of $\{\exp t \xi\}$ and $M, M \cap \Gamma$ is a lattice in $M$, and the $\tilde{U}$-action on $M$ is trivial.

Proof. Since $\mathfrak{N}$ is generated by $\xi$ and $\eta$ it follows that $\mathfrak{N} /[\mathfrak{N}, \mathfrak{N}]$ is a two dimensional vector space spanned by the images of $\xi$ and $\eta$. Since $\mathfrak{U}(\xi)=\xi+\eta$ this implies that $\langle\eta\rangle+[\mathfrak{N}, \mathfrak{N}]$ is a $\mathfrak{U}$-invariant Lie ideal in $\mathfrak{N}$. Let $\mathfrak{M}=\langle\eta\rangle+[\mathfrak{N}, \mathfrak{N}]$ and $M$ be the simply connected subgroup of $N$ corresponding to $\mathfrak{M}$. Then $M$ is a $(d-1)$-dimensional $\tilde{U}$-invariant subgroup and $N$ is the semidirect product of $\{\exp t \xi\}$ and $M$. Also, since $[N, N] \cap \Gamma$ is a lattice in $[N, N]$ and $M /[N, N]$ is the set of fixed points of the factor of $\tilde{U}$ on $N /[N, N]$ it follows that $M \cap \Gamma$ is a lattice in $M$. We conclude the proof by showing that $M$ is abelian and the $\tilde{U}$ action on $M$ is trivial.

For all $t \in \mathbb{R}$ let $x_{t}=\exp t \xi$. Then as seen before, for every $t \in \mathbb{R}, x_{t} M \Gamma / \Gamma$ is $U$-invariant and when it is viewed as $M /(M \cap \Gamma)$ the $U$-action on $x_{t} M \Gamma / \Gamma$, $t \in \mathbb{R}$ corresponds to the affine automorphism induced by $T_{a_{t}} \circ \tilde{U}$, where $a_{t}=$ $x_{t}{ }^{-1} \tilde{U}\left(x_{t}\right)=\exp (-t \xi) \exp (t(\xi+\eta))$. Let $L=M /[M, M]$ and $\Delta=[M, M](M \cap$ $\Gamma)$. Let $\mathfrak{L}$ be the Lie algebra of $L$ and $\tau: \mathfrak{L} \rightarrow \mathfrak{L}$ be the map defined by $\tau(\mu+[\mathfrak{M}, \mathfrak{M}])=[\xi, \mu]+[\mathfrak{M}, \mathfrak{M}]$ for all $\mu \in \mathfrak{M}$. Since $\mathfrak{N}$ is generated by $\xi$ and $\eta$ it follows that $\mathfrak{L}$ is spanned by $\left\{\eta, \tau(\eta), \ldots, \tau^{n-1}(\eta)\right\}$, where $n$ is the least positive integer such that $\tau^{n}(\eta)=0$, and furthermore the set forms a basis of $\mathfrak{L}$. 
Now $N /[M, M]$ may be viewed as the semidirect product of the one-parameter subgroup $\Phi:=\left\{x_{t}\right\}$ and $\mathfrak{L}$, identifying $L$ with $\mathfrak{L}$ canonically. In turn the group may be realised as a group of $(n+1) \times(n+1)$ matrices, with $x_{t}$ represented by $\left(\begin{array}{cc}\exp t \nu & 0 \\ 0 & 1\end{array}\right)$ where $\nu=\left(\nu_{i j}\right)$ is the $n \times n$ matrix given by $\nu_{i j}=1$ if $j=i+1$ and 0 otherwise, and the elements of $\mathfrak{L}$ are represented by $\left(\begin{array}{cc}I_{n} & v \\ 0 & 1\end{array}\right)$ with $I_{n}$ the $n \times n$ identity matrix and $v$ a column vector ${ }^{t}\left(v_{1}, \ldots, v_{n}\right)$; specifically the entries $v_{1}, \ldots, v_{n}$ are given by the coordinates of the element with respect to the basis $\left\{\tau^{n-1}(\eta), \ldots, \eta\right\}$ (in that order). From this the image of $a_{t}$ in $L$ can be computed explicitly to be $\sum_{k=0}^{n-1} c_{k} t^{k+1} \tau^{k}(\eta)$, where $c_{k}=(-1)^{k} / k$ ! for all $k$.

We can find $t$ such that $1, c_{0} t, c_{1} t^{2}, \ldots c_{n-1} t^{n}$ are linearly independent over $\mathbb{Q}$. This is because all $c_{k}$ being nonzero, the set of $t$ for which the elements are linearly dependent is countable. For $t$ satisfying this condition, as discussed above, the action $T_{a_{t}} \circ U$ on $L / \Delta$ is minimal, and in turn the $T_{a_{t}} \circ U$ action on $M /(M \cap \Gamma)$ is minimal. Since it is almost automorphic by Corollary 1.2 that $M$ is abelian and the $\tilde{U}$ action on $M$ is trivial.

\section{Miscellania}

In this section we discuss the issue in a broader context. Firstly we prove the following result which extends the scope of applicability of the results of the earlier sections.

We shall say that a homeomorphism $\varphi$ of a locally compact second countable space $X$ admits a convergent trajectory if there exists $x \in X$ such that $\varphi(x) \neq x$ and either $\left\{\varphi^{k}(x)\right\}_{k=0}^{\infty}$ or $\left\{\varphi^{-k}(x)\right\}_{k=0}^{\infty}$ converges in $X$, as $k \rightarrow \infty$; we note that in either case the limit is a fixed point of $\varphi$. Clearly, if $\varphi$ admits a convergent trajectory, or more generally if a factor of $\varphi$ admits a convergent trajectory, then $\varphi$ is not almost automorphic. This means in particular that if $T$ as in the hypothesis of the next proposition is also assumed to be almost automorphic, then only the second alternative as in the conclusion can hold.

Proposition 6.1. Let $N$ be a simply connected nilpotent Lie group and $\Gamma$ be a lattice in $N$. Let $T=T_{a} \circ A$ be an affine automorphism of $N / \Gamma$, where $a \in N$ 
and $A$ is an automorphism of $N / \Gamma$. Let $\tilde{A}$ be the automorphism corresponding to $A$. Then one of the following holds:

i) there exists a closed connected normal $\tilde{A}$-invariant subgroup $M$ of $N$ such that $M \Gamma$ is closed, and the factor $\bar{T}$ of $T$ on $N / M \Gamma$ admits a convergent trajectory.

ii) there exists $r \in \mathbb{N}$ such that $\tilde{A}^{r}$ is unipotent.

Proof. Firstly consider the case when $N=\mathbb{R}^{d}$, for some $d \geq 1$. Let $V$ be the largest $\tilde{A}$-invariant subspace of $\mathbb{R}^{d}$ on which 1 is the only eigenvalue, and $W=\mathbb{R}^{d} / V$. Since $\tilde{A}$ leaves $\Gamma$ invariant, it follows that the image $\Delta=(V+\Gamma) / \Gamma$ of $\Gamma$ in $W$ is a lattice in $W$. It suffices to prove the proposition for the factor of $T$ on $R / W$ and hence without loss of generality we may assume that $V$ is trivial and $W=\mathbb{R}^{d}$; that is, 1 is not an eigenvalue of $\tilde{A}$. The last condition implies, via simple linear algebra, that $T_{a} \circ \tilde{A}$ has a fixed point $b \in \mathbb{R}^{d}$. Then $T_{a} \circ \tilde{A}=T_{b} \circ \tilde{A} \circ T_{b}^{-1}$. If $\tilde{A}$ has an eigenvalue of absolute value different from 1 then $\tilde{A}$ admits a convergent trajectory and hence so does $T_{a} \circ \tilde{A}$, and so statement (i) holds in this case. Now suppose that all the eigenvalues of $\tilde{A}$ are of absolute value 1 . Since $\tilde{A}$ leaves $\Gamma$ invariant this further implies that all the eigenvalues are roots of unity; (one shows this by considering the characteristic polynomials of $\tilde{A}^{k}, k \in \mathbb{N}$, and showing that under the given condition their roots, which are eigenvalues of $\tilde{A}^{k}$, would belong to a fixed finite set). Hence there exists $r \in \mathbb{N}$ such that $\tilde{A}^{r}$ is unipotent. This proves the proposition when $N$ is abelian.

Now consider the general case. Let $\bar{A}$ denote the factor of $\tilde{A}$ on $N /[N, N]$. Suppose that alternative (i) does not hold. From the special case we then get that there exists $r \in \mathbb{N}$ such that $\bar{A}^{r}$ is unipotent. Therefore in proving the proposition we may without loss of generality assume that $\bar{A}$ is unipotent. By an argument well-known to Lie group theorists this implies that $\tilde{A}$ is also unipotent. Let us recall the argument here, briefly, for the reader's convenience: Let $\mathfrak{N}$ be the Lie algebra of $N$ and $\mathfrak{M}$ be the largest $d \tilde{A}$-invariant subspace on which $d \tilde{A}$ is unipotent. It can be seen that $\mathfrak{M}$ is a Lie subalgebra of $\mathfrak{N}$, and as $\bar{A}$ is unipotent we get $\mathfrak{M}+[\mathfrak{N}, \mathfrak{N}]=\mathfrak{N}$. Substituting from the equation successively we get $\mathfrak{N}=\mathfrak{M}+[\mathfrak{M}+[\mathfrak{N}, \mathfrak{N}], \mathfrak{M}+[\mathfrak{N}, \mathfrak{N}]] \subseteq \mathfrak{M}+[\mathfrak{N},[\mathfrak{N}, \mathfrak{N}]] \subseteq \cdots \subseteq \mathfrak{M}$, since $\mathfrak{N}$ is nilpotent. Thus $\mathfrak{N}=\mathfrak{M}$ which shows that $\tilde{A}$ is unipotent. This completes the proof of the proposition.

Remark 6.2. We recall that a homeomorphism $\varphi$ of a locally compact second 
countable space $X$ is said to be distal if for $x, y \in X, x \neq y$, there does not exist any sequence $\left\{k_{i}\right\}$ in $\mathbb{Z}$ such that $\varphi^{k_{i}}(x)$ and $\varphi^{k_{i}}(y)$ converge to the same point as $i \rightarrow \infty$. Proposition 6.1 also shows that if an affine automorphism $T=T_{a} \circ A$, as in the statement of the proposition, is assumed to be distal then $A^{r}$ is unipotent for some $r \in \mathbb{N}$; in other words, the automorphism part of $T^{r}$ is unipotent. Related general results for distal group actions by group automorphisms, with analogous conclusions about the action of a subgroup of finite index, may be found in [1]. The preceding conclusion can indeed be deduced, albeit not directly, from the results of [1]. On the other hand Proposition 6.1 provides a simple and direct argument in the case at hand.

We conclude with the following remark.

Remark 6.3. The notion of almost automorphic homeomorphisms extends naturally to group actions $G \times X \rightarrow X$, where $G$ is a group acting by homeomorphisms of $X$ : the action is almost automorphic if for any sequence $\left\{g_{i}\right\}$ in $G$ and $x, y \in X$ if $g_{i} x \rightarrow y$ then $g_{i}^{-1} y \rightarrow x$. Clearly when an action is almost automorphic then the homeomorphism corresponding to the action of any individual element is almost automorphic. It seems to us however that the converse of this is not true. This would follow if it is proved, which also we expect to be true, that any group of automorphism of a compact nilmanifold whose action is almost automorphic is necessarily abelian; (it can be readily seen that there exist nonabelian subgroups of $S L(4, \mathbb{Z})$ all whose elements are unipotent elements of nilrank at most 1 , so the action of each of them on $\mathbb{T}^{4}$ is almost automorphic (by Proposition [5.1)).

Acknowledgement: The first and third named author would like to thank the School of Physical Sciences (SPS), Jawaharlal Nehru University (JNU), New Delhi, India for hospitality while some of the work was done. The third named author would also like to thank the National Board of Higher Mathematics (NBHM), DAE, Govt. of India for a post-doctoral fellowship. The second named author would like to thank the School of Mathematics, Tata Institute of Fundamental Research (TIFR), Mumbai, India for hospitality while some of the work was done. 


\section{References}

[1] H. Abels. Distal affine transformation groups. J. reine angew. Math. 299/300 (1978), 294-300.

[2] W. Parry, Ergodic properties of affine transformations and flows on nilmanifolds, Amer. J. Math. 91 (1969), 757-771.

[3] M.S. Raghunathan, Discrete Subgroups of Lie groups, Springer, 1972.

[4] P. Walters, An Introduction to Ergodic Theory, Graduate Texts in Mathematics, 79, Springer, 1982.

S.G. Dani

Department of Mathematics

Indian Institute of Technology Bombay

Powai, Mumbai 400076

India

shrigodani@gmail.com

Riddhi Shah

School of Physical Sciences

Jawaharlal Nehru University

New Delhi 110067

India

riddhi. kausti@gmail.com

Puneet Sharma

COE - Systems Science

Indian Institute of Technology Jodhpur

Residency Road, Ratanada

Jodhpur - 342011

India

puneet8111.iitd@gmail.com 